\title{
Contact Force Mediated Rapid Deposition of Colloidal Microspheres Flowing over Microstructured Barriers
}

\author{
P. Prakash,* A. Z. Abdulla, and M. Varma*
}

Cite This: Langmuir 2021, 37, 6915-6922

Read Online

ABSTRACT: Deposition of particles while flowing past constrictions is a ubiquitous phenomenon observed in diverse systems. Some common examples are jamming of salt crystals near the orifice of salt shakers, clogging of filter systems, gridlock in vehicular traffic, etc. Our work investigates the deposition events of colloidal microspheres flowing over microstructured barriers in microfluidic devices. The interplay of DLVO, contact, and hydrodynamic forces in facilitating rapid deposition of microspheres is discussed. Noticeably, a decrease in the electrostatic repulsion among microspheres leads to linear chain formations, whereas an increase in roughness results in rapid deposition.

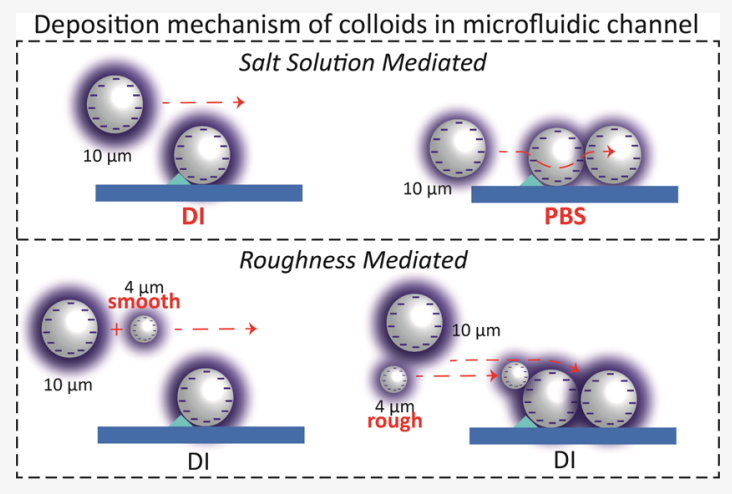

\section{INTRODUCTION}

A deposition process is a gradual buildup of heaps of cellular or particle milieu over time. It usually entails the simultaneous action of DLVO, contact, and hydrodynamic forces. ${ }^{1}$ The dominant physical force promoting deposition is system dependent. For example, the aggregation of colloidal particles is driven by DLVO forces, ${ }^{2}$ jamming events are supported by contact force, ${ }^{3}$ and active crystals can be formed entirely due to hydrodynamic interactions. ${ }^{4,5}$ Besides, there are other frequently ignored phenomena such as many-body interaction, tribological effects, etc. ${ }^{6-8}$ The understanding of deposition processes is also highly relevant for various industrial processes. Several technologies rely on suppression of clogging in porous channels such as membrane filters, deep-bed filtration technology, inkjet printing, porous catalyst pellets, etc.

Competing physical phenomena act at once, making it exceptionally challenging to pin down the dominant mechanism during deposition events. Microfluidic devices have long been used to decipher several aspects of the deposition process. The channel geometry is one of the most explored aspects of clogging, with numerous studies on the role of connectivity, tortuosity, constrictions, and porosity. ${ }^{9-15}$ Several numerical studies as well have guided the experiments by proposing clogging-phase diagrams. ${ }^{16,17}$ The simplest form of clogging occurs during pore blockage by particles whose size is larger than the pore size. ${ }^{18}$ However, clogging is common, even when the size of flowing particles are much smaller than the pore size. At low volume fractions $(\sim 1 \% \mathrm{w} / \mathrm{v})$, the collective effects are negligible and clogging proceeds as a single particle deposition process, whereas at high volume fractions $(\sim 20 \% \mathrm{w} / \mathrm{v})$, clogging is influenced by the collective behavior of many interacting particles. ${ }^{19-21}$ The three major physical interactions in a deposition process are particle-fluid, particle-particle, and particle-surface, typically tuned by varying the flow rate and ionic strength. ${ }^{22-25}$

There is very little control on the spatial location where the clogging occurs in a microfluidic channel, making it tricky to study deposition events at a single particle level. We bypass this problem by fabricating microstructures over glass substrates, allowing precise deposition of the first particle. ${ }^{26}$ The deposition process is then explored using the first particle as a seed. Interestingly, colloidal microspheres form a linear chain at low ionic strength, whereas a slight increase in the roughness of microspheres leads to rapid deposition. The role of roughness in the deposition process is not understood and so far has been explored only in the context of shear-thickening of colloidal suspensions. ${ }^{27}$ Our work lays out the physical and chemical conditions required for rapid deposition of colloidal microspheres, giving valuable insights into the clogging phenomena. Further, the size of microspheres $(10+4 \mu \mathrm{m})$ used is very similar to the size of the cellular milieu (neutrophils: $12-14 \mu \mathrm{m}$, platelets: $2-3 \mu \mathrm{m}$, RBCs: $6-8$ $\mu \mathrm{m})$ in arteries $^{28}$ and are reminiscent of the arterial plaque formation in arteries leading to heart attack. ${ }^{29-31}$

Received: February 7, 2021

Revised: May 16, 2021

Published: June 2, 2021 


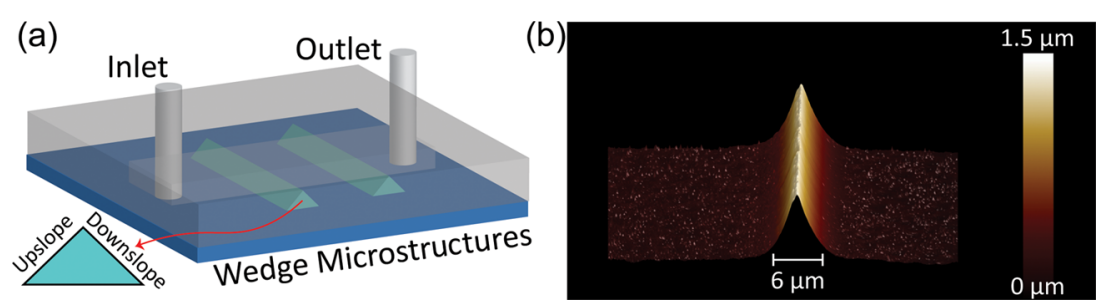

Figure 1. (a) Schematic of a PDMS microfluidic device $(h=100 \mu \mathrm{m}, w=500 \mu \mathrm{m})$ affixed over wedge microstructures. Flow is driven from the inlet to the outlet, first encountering the upslope and then downslope. (b) Atomic force microscopy (AFM) of the microstructures $(h=1.5 \mu \mathrm{m}, w$ $=6 \mu \mathrm{m})$.
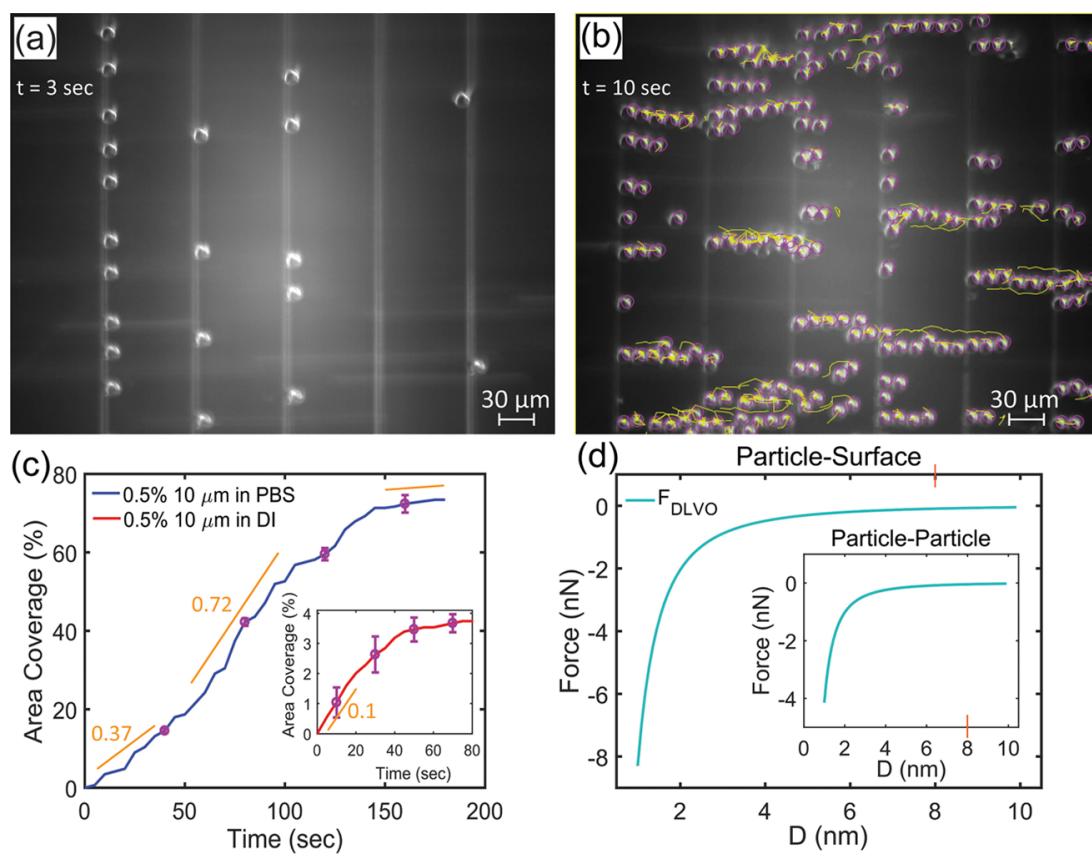

Figure 2. Deposition of $10 \mu \mathrm{m}$ microspheres at a flow rate of $10 \mu \mathrm{L} / \mathrm{min}$. (a) Microspheres in the presence of DI water pin on the downslope of the wedge microstructures. (b) Microspheres in the presence of $0.1 \mathrm{M}$ phosphate-buffered saline (PBS) deposit in the form of linear chains. (c) The fractional area coverage of microspheres flowing in $0.1 \mathrm{M}$ PBS vs time. The curve saturates after $\sim 2 \mathrm{~min}$. Inset figure shows the area coverage when microspheres pin only on the downslope in the presence of DI water. (d) DLVO force between microspheres of size $10 \mu \mathrm{m}$ and the glass substrate in DI water medium. Inset image shows the DLVO force between two microspheres of size $10 \mu \mathrm{m}$ in DI water medium.

\section{MATERIALS AND METHODS}

The deposition of polystyrene microspheres of size $10 \mu \mathrm{m}$ (density = $1.05 \mathrm{~g} / \mathrm{mL}$ ) is studied by flowing them over wedge-like fabricated structures in a microfluidic device (Figure 1a). The wedge microstructures are fabricated by patterning a $2.2-\mu \mathrm{m}$-thick and 5$\mu$ m-wide photoresist (S1813) in an array over glass substrates. Subsequently, the resist patterned substrate is isotropically etched using a hydrofluoric acid ( $\mathrm{HF})$-based etchant $(10 \% \mathrm{HF}+7 \% \mathrm{HCl})$ for $15 \mathrm{~s}$. This process yields wedge-shaped symmetric microstructures of height $\sim 1 \mu \mathrm{m}$ and width $\sim 6 \mu \mathrm{m}$ (Figure 1b). ${ }^{26}$ A poly(dimethylsiloxane) (PDMS) microfluidic device of height $100 \mu \mathrm{m}$ and width $500 \mu \mathrm{m}$ is then clamped on the microstructured substrate to seal the channel. The device is imaged at $30 \mathrm{fps}$ by a custom-built inverted CMOS camera (Thorlabs) assembly illuminated using ringshaped light (Supporting Information (SI), Figure 1a). Four repeats were carried out in each case to study the deposition trends across different scenarios. Before each repetition, the substrate was washed with the piranha solution $\left(3 \mathrm{H}_{2} \mathrm{SO}_{4}+\mathrm{H}_{2} \mathrm{O}_{2}\right)$ to maintain its pristine condition.

\section{RESULTS AND DISCUSSION}

Pinning of Microspheres in a Deionized (DI) Water Medium. The colloidal microspheres of size $10 \mu \mathrm{m}(0.5 \% \mathrm{w} /$ $\mathrm{v}$ ) in deionized ultrafiltered water (DI water) selectively pin on the downslope (Figure 1a) side of the microstructures when passed at a flow rate of $10-50 \mu \mathrm{L} / \mathrm{min}$ (Figure 2a, Video S1). For lower flow rates (i.e., $<10 \mu \mathrm{L} / \mathrm{min}$ ), the microspheres also pin on the upslope in addition to the downslope due to their inability in crossing the upslope gravitational potential barrier (Video S2). Thus, $10 \mu \mathrm{L} / \mathrm{min}$ is the lowest flow rate with no upslope pinning. ${ }^{26}$ For higher flow rates we still do not see upslope pinning; however, the rate of pinning drops drastically (SI, Figure S1b). Due to the high pinning rate and precise downslope pinning, we initiated all the subsequent experiments at a flow rate of $10 \mu \mathrm{L} / \mathrm{min}$.

The horizontal drag force on microspheres at a flow rate of $10 \mu \mathrm{L} / \mathrm{min}$ is $0.12 \mathrm{nN}$ regardless of pinning on the upslope or downslope region as estimated from the hydrodynamic simulation (Section S2) performed using "COMSOL Multiphysics". ${ }^{32}$ In contrast, microspheres experience a lift force $(11.7 \mathrm{pN})$ on the upslope whereas a downward thrust $(-13.5$ $\mathrm{pN}$ ) on the downslope, explaining the preferential pinning on the downslope. Interestingly, microspheres do not pin beyond a flow rate of $50 \mu \mathrm{L} / \mathrm{min}$ (drag force $\sim 0.6 \mathrm{nN}$ ); however, once pinned (i.e., at lower flow rates $\leq 50 \mu \mathrm{L} / \mathrm{min}$ ), they do not detach even at a very high flow rate of $300 \mu \mathrm{L} / \mathrm{min}$, 


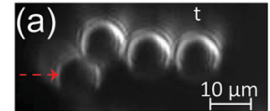

(b)

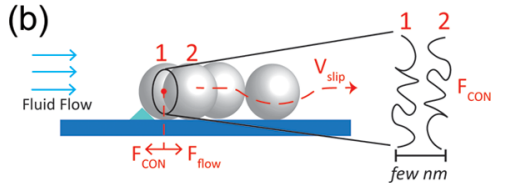

(c)

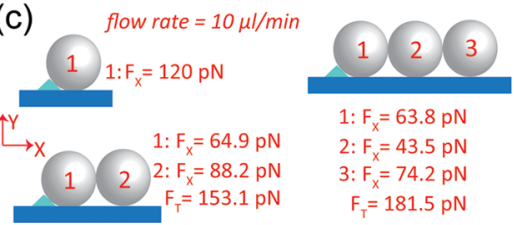

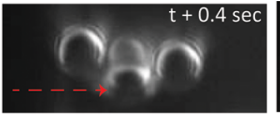

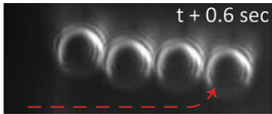

(d)

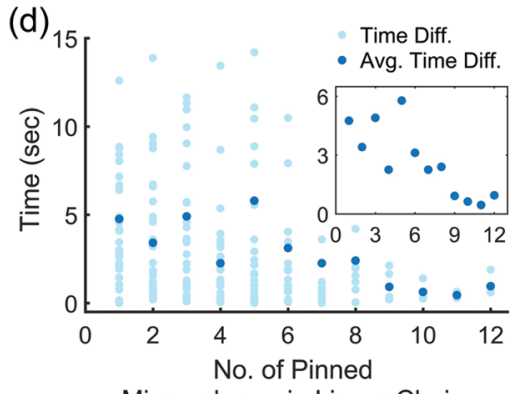

Microspheres in Linear Chain

Figure 3. Linear chain formation in a $10 \mu \mathrm{m}$ microsphere when flown in $0.1 \mathrm{M}$ PBS at a flow rate of $10 \mu \mathrm{L} / \mathrm{min}$. (a) Sequence of images showing growth of the chain from 3 to 4 microspheres. (b) Microsphere grazes through the chain and deposits in the front. (c) Force redistributes along the chain such that on average the drag force increases by $\sim 30 \mathrm{pN}$ per microsphere. (d) Time difference between the pinning of successive microspheres evaluated over all of the linear chains. Inset image shows a drop in the average time difference as the length of the chain grows.

experiencing a shear drag force of $\sim 4.9 \mathrm{nN}$. This suggests that over time microspheres settle to a position of higher local stability. It is possible that the surface of microspheres and glass substrate realign, leading to increased frictional contact area and adhesion. The microspheres remain pinned even if the device is flipped upside down, suggesting a role of the attractive DLVO force as well. Below, we have explored the deposition rate by introducing salt solution to enhance the attractive DLVO force and microspheres of higher roughness to increase the frictional force.

Linear Chain Formation in the Presence of Salt Medium. Microspheres at a flow rate of $10 \mu \mathrm{L} / \mathrm{min}$ and in DI water medium selectively pin on the downslope. ${ }^{26}$ The same $10 \mu \mathrm{m}$ microspheres $(0.5 \% \mathrm{w} / \mathrm{v})$, when flown in $0.1 \mathrm{M}$ phosphate buffer saline (PBS), form linear chains (Video S3, Figure $2 \mathrm{~b})$. At higher PBS concentration $(1 \mathrm{M})$, the colloidal solution is unstable and readily forms aggregates, whereas, at a lower concentration $(0.01 \mathrm{M})$, the behavior is similar to single pinning events seen in DI water medium. The chain formation in the $0.1 \mathrm{M}$ PBS medium proceeds by pinning of single microspheres on the downslope, which then acts as a seed for successive microspheres to deposit one by one, growing into a linear chain (Video S3). The microstructure facilitates pinning of only the first particle; from thereon, the dynamics of the chain formation is governed by particle-particle interaction. Close contact between the incoming and already pinned microspheres is crucial for the microspheres to glide their way to form linear chains.

We define the fractional area coverage as the ratio of space occupied by microspheres to the total space on the substrate (Figure 2c). The space occupied by a microsphere is assumed to be a square of side length equivalent to the microsphere diameter as it becomes unavailable for the next incoming microsphere. The fractional area coverage in DI water (inset Figure 2c) saturates over time due to the filling of all available pinning sites, i.e., microstructures. In the presence of PBS (Figure 2c), microspheres grow into linear chains (Video S3), thereby propelling the area coverage up to $70 \%$ (SI, Figure S4a). The microsphere flown in DI water pins only on microstructures, and hence the area of coverage saturates quickly, whereas it takes longer for the area coverage to saturate in PBS $(0.1 \mathrm{M})$ due to the growth of microspheres into linear chains.
The interplay among hydrodynamics, DLVO $\left(F_{\mathrm{DLVO}}\right)$, and contact forces is responsible for this fascinating linear alignment of microspheres. The DLVO force comprises electrostatic double-layer repulsion $\left(F_{\mathrm{EDL}}\right)$ and attractive van der Waals force $\left(F_{\mathrm{VDW}}\right)$. The electrostatic potential of the microspheres determined by $\zeta$-potential measurement drops from $-31.5 \pm 1.7 \mathrm{mV}$ in DI water to $-4.33 \pm 0.86 \mathrm{mV}$ in 0.1 M PBS. The corresponding Debye length $(\sim 0.3 / \sqrt{[\mathrm{C}]} \mathrm{nm}$, $\mathrm{C}$-buffer concentration in M) drops from $948.7 \mathrm{~nm}$ in DI water to $\sim 1 \mathrm{~nm}$, respectively. ${ }^{33}$ The decrease in electrostatic potential and Debye length in the PBS medium leads to lower repulsion $\left(F_{\mathrm{EDL}}\right)$ and therefore increases the probability of contact, allowing the microspheres to graze closely, resulting in a serial deposition. The attractive van der Waals component of the DLVO force relies on the gap between the microspheres and the substrate, which is set by the roughness of the interacting surfaces. ${ }^{34}$ The roughness of the glass substrate $R_{\mathrm{a}}$ is $\sim 8.0 \mathrm{~nm}$ (Figure $4 \mathrm{~b}$ ), which sets a lower limit on the minimum grazing distance with the microspheres. The drag force at a flow rate of $10 \mu \mathrm{L} / \mathrm{min}$ on the very first pinned particle in the linear chain is $F_{\text {flow }}=0.12 \mathrm{nN}$ (Section S2). The attractive DLVO force between the microsphere and the glass substrate at a gap of $8 \mathrm{~nm}$ in DI water is $F_{\mathrm{DLVO}} \approx 0.09 \mathrm{nN}$ (Figure 2d; see Section S3 for the DLVO force calculations), which is insufficient to counter the drag by fluid flow. It is likely that the microsphere arrests due to the collective effects of both DLVO force and frictional force.

The stick and slip motion exhibited by the second incoming microspheres onward in the presence of the PBS medium (Video S3, Figure 3a) is a characteristic feature of frictional force. ${ }^{35-39}$ We define contact force $\left(F_{\text {con }}\right)$ as the total frictional force due to attractive DLVO interaction and the surface roughness acting against the drag force to arrest the microspheres. The contact force while the microsphere slips can be estimated as follows

$$
F_{\text {con }}=F_{\text {flow }}-F_{\text {slip }}
$$

where $F_{\text {flow }}=0.12 \mathrm{nN}$ is the drag force on a static microsphere in the presence of flow, and $F_{\text {slip }}$ is the drag force when the microspheres are slipping against the pinned microspheres at a speed " $V_{\text {slip }}$ ". The drag force on a particle grazing near a surface can be analytically derived as $1.7 \times F_{\text {Stokes }}$ (Section S4), where $F_{\text {Stokes }}=6 \pi \eta \mathrm{rV}$ is the Stokes drag on a sphere moving with 

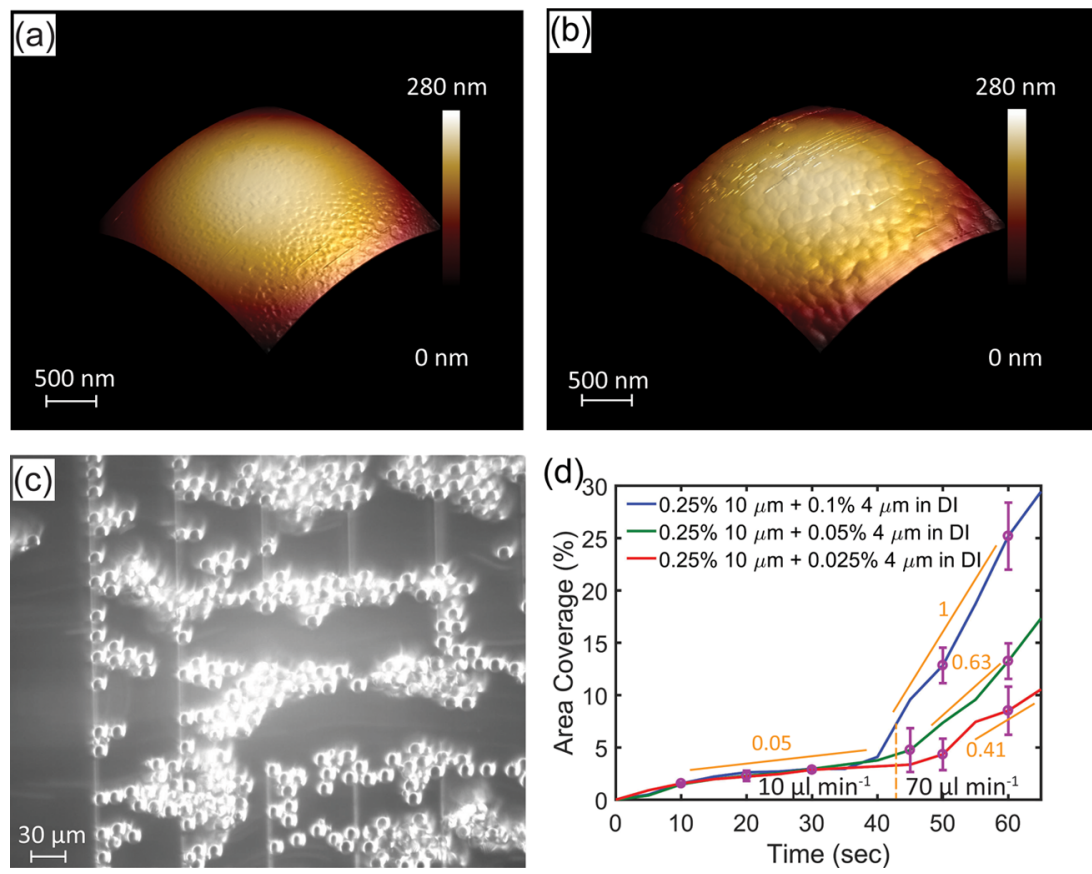

Figure 4. Deposition of multi-microspheres $(4+10 \mu \mathrm{m})$ over wedge microstructures. (a) AFM of $4 \mu \mathrm{m}$ smoother microspheres of roughness $R_{\mathrm{a}}=$ $1.5 \mathrm{~nm}$. (b) AFM of $4 \mu \mathrm{m}$ rough microspheres $\left(R_{\mathrm{a}}=4.7 \mathrm{~nm}\right)$. (c) Rapid deposition of microspheres $(4+10 \mu \mathrm{m})$ at a high flow rate of $70 \mu \mathrm{L} / \mathrm{min}$. (d) The rate of area coverage jumps up to 20 -fold as the flow rate is increased from 10 to $70 \mu \mathrm{L} / \mathrm{min}$.

speed " $V$ " $\left(\eta=8.9 \times 10^{-4} \mathrm{~Pa} \cdot \mathrm{s}\right.$ is the viscosity of water and $r=$ $5 \mu \mathrm{m}$ is the radius of the microspheres). ${ }^{40-44}$ The average slip velocity " $V_{\text {slip }}=68.8 \pm 8.4 \mu \mathrm{m} / \mathrm{s}$ " is estimated by taking the ratio of the distance covered by the microspheres while slipping through the linear chain (Figure 3a) and corresponding time consumed (yellow lines tracked using ImageJ in Figure $2 b$ ). The force required for a microsphere to attain the slip velocity can be approximated as $F_{\text {slip }}=1.7 \times 6 \pi \eta r V_{\text {slip }}=9.8$ $\mathrm{pN}$. The contact force $F_{\mathrm{CON}}=F_{\text {flow }}-F_{\text {slip }}=0.12 \mathrm{nN}-9.8 \mathrm{pN}$ $\approx 0.11 \mathrm{nN}$ (schematic shown in Figure $3 \mathrm{~b}$ ) is marginally lower than the drag due to fluid flow which is essential for the slipping to occur. The incoming microspheres graze through the linear chain and eventually deposit on the posterior end guided by fluid flow. Using the fluid flow simulation discussed in Section S2, we estimated the drag force on the linear chain as it grows. The drag force on the very first microsphere at a fluid flow rate of $10 \mu \mathrm{L} / \mathrm{min}$ is $120 \mathrm{pN}$ and increases on an average by only $30 \mathrm{pN}$ per microsphere (Figure $3 \mathrm{c}$ ). Therefore, as the chain grows, the average drag force per microsphere redistributes to a lower value than the drag on the first microsphere. Hence, an increase in the length of chain does not adversely affect its stability.

The time difference between the deposition of successive microspheres is shown for all linear chains in Figure 3d. We can see a trend of drop in average time difference as the length of the chain grows (inset Figure 3d). The time consumed by the microspheres to deposit is a function of the total number of microspheres arriving and their probability of deposition. Since there is no physical or chemical change in the system, the probability of deposition is expected to be nearly the same. A marginal increase in the drag force is anticipated as the deposition of microspheres reduces the cross-sectional area of the flow channel, resulting in an increased mean fluid velocity and, consequently the drag force.

A closer look at the tracks shown in Video S4 (longer version of Video S3) suggests a significant coupling among nearby chains. As the length of the chain grows, microspheres jump from preceding chains to the forward-forming chains, possibly increasing the rate of incoming microspheres. In contrast, deposition of the first few microspheres is statistical as the incoming microspheres interact with a chain only if they happen to lie in their flow direction. Additionally, some chains are not entirely straight and have microspheres protruding out, which act as extra seeds for the incoming microsphere to latch on and glide their way to deposit in the front.

Rapid Deposition in the Presence of Rough Microspheres. Next, we used microspheres with different surface roughness values to better understand the role of friction force. A mixture of 10 and $4 \mu \mathrm{m}$ microspheres of two different surface roughness values were used. We procured $4 \mu \mathrm{m}$ microspheres of roughness $1.5 \mathrm{~nm}$ (Figure $4 \mathrm{a}$ ) and $4.7 \mathrm{~nm}$ (Figure $4 \mathrm{~b}$ ), respectively (Section S5). A mixture of $0.1 \% \mathrm{w} / \mathrm{v}$ $4 \mu \mathrm{m}$ microspheres of lower roughness $\left(R_{\mathrm{a}}=1.5 \mathrm{~nm}\right)$ and $0.25 \% \mathrm{w} / \mathrm{v} 10 \mu \mathrm{m}$ microspheres, when flown in DI medium, did not affect the deposition, and only $10 \mu \mathrm{m}$ microspheres were pinned as before (Figure 2a, Section S6). In contrast, rapid deposition of microspheres is observed when the same mixture composition but with $4 \mu \mathrm{m}$ microspheres of higher roughness $\left(R_{\mathrm{a}}=4.7 \mathrm{~nm}\right)$ is flown (Video S5). The deposition of the first few microspheres is shown in the close-up movie Video S6. The $4 \mu \mathrm{m}$ microspheres of lower roughness can approach the $10 \mu \mathrm{m}$ microspheres from a closer distance and are likely to have a higher DLVO force as compared to the rougher $4 \mu \mathrm{m}$ microspheres. However, the occurrence of rapid deposition only in the presence of the rough $4 \mu \mathrm{m}$ microspheres suggests a dominant role of friction force in the arrest of the microspheres.

Initially, multi-microspheres $(4+10 \mu \mathrm{m})$ in DI water are flown at the rate of $10 \mu \mathrm{L} / \mathrm{min}$; pinning microspheres of size $10 \mu \mathrm{m}$ in the downslope region, whereas microspheres of size 4 $\mu \mathrm{m}$ pass through. The pinned microspheres then act as seeds for rapid deposition at a higher flow rate of $70 \mu \mathrm{L} / \mathrm{min}$. Rapid 


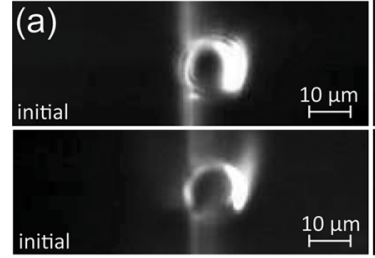

(b)

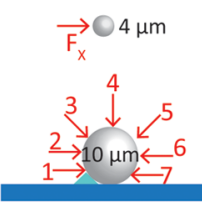

(c)

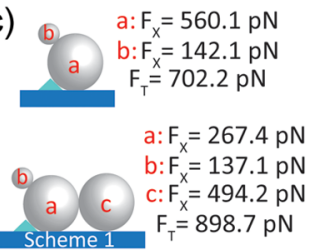

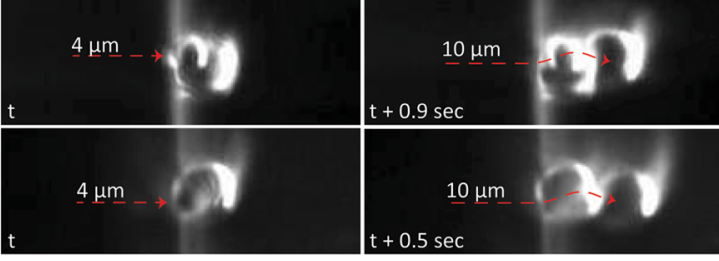

(d)

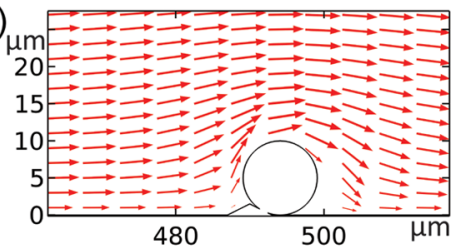

(e)

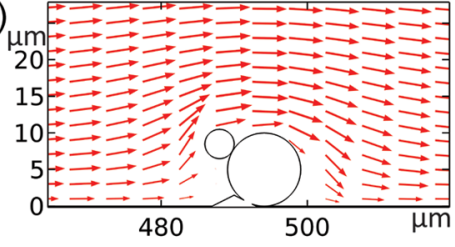

Figure 5. Hydrodynamic drag on $4 \mu \mathrm{m}$ microspheres pinned over $10 \mu \mathrm{m}$ microspheres at a flow rate of $70 \mu \mathrm{L} / \mathrm{min}$. (a) Image series showing subsequent deposition of the $4 \mu \mathrm{m}$ microspheres and $10 \mu \mathrm{m}$ microspheres on the already pinned $10 \mu \mathrm{m}$ microspheres. (b) Hydrodynamic drag force on the $4 \mu \mathrm{m}$ microsphere at different positions. Surprisingly, positions around 3 facing the maximum hydrodynamic drag are preferred positions of pinning as seen in the videos. (c) Increase in drag force due to deposition of the incoming $10 \mu \mathrm{m}$ microsphere on the 10 and $4 \mu \mathrm{m}$ microsphere system. Microspheres face higher drag in Scheme 2 and glide to Scheme 1 configuration, where they face a much lower drag. (d) Flow field over the $10 \mu \mathrm{m}$ microspheres. (e) Flow field over the 10 and $4 \mu \mathrm{m}$ microsphere system.

deposition proceeds by first pinning of $4 \mu \mathrm{m}$ rough microspheres on the already pinned $10 \mu \mathrm{m}$ microspheres, and further advances when the incoming $10 \mu \mathrm{m}$ microspheres deposit by latching onto $4 \mu \mathrm{m}$ microspheres. Interestingly, the rapid deposition of microspheres does not occur below a flow rate of $70 \mu \mathrm{L} / \mathrm{min}$. The deposition in the multi-microsphere system $(10+4 \mu \mathrm{m}$ in DI water) grows in three dimensions (Figure 4c) as opposed to the linear chains in the lonemicrosphere system (10 $\mu \mathrm{m}$ in PBS) previously discussed (Figure 2b).

The rate of single pinning events of $10 \mu \mathrm{m}$ microspheres decreases by a factor of 2 from 0.1 to 0.05 as the concentration of microspheres is halved from $0.5 \% \mathrm{w} / \mathrm{v}$ (inset Figure $2 \mathrm{c}$ ) to $0.25 \% \mathrm{w} / \mathrm{v}$ (Figure 4d). Accordingly, we represent the area coverage $(\mathrm{AC})$ in time " $t$ " as

$$
\mathrm{AC}=\delta \rho t
$$

where " $\delta$ " is the sticking probability and " $\rho$ " is the volume fraction of the $10 \mu \mathrm{m}$ microspheres. The magnitude of sticking probability $(\delta)$ is a function of the DLVO interaction, contact force, and flow rate. If the flow rate is kept constant, then the sticking probability relies only on material properties such as salt concentration and roughness. For instance, the slope of AC vs time while keeping the concentration $(\rho)$ fixed increases from 0.1 in DI water to 0.37 in PBS medium (Figure 2c). This increase is attributed to the reduction in the Debye length (PBS medium leads to lower electrostatic repulsion) resulting in an increased sticking probability. In the case of a multimicrosphere system $(10+4 \mu \mathrm{m})$ in DI water, the deposition rates are even higher, where the area coverage rate increases up to 20 -folds as the flow rate is increased to $70 \mu \mathrm{L} / \mathrm{min}$ (Figure $4 \mathrm{~d})$. The AC was calculated directly by thresholding the images using ImageJ to construct the outer border of deposited microspheres. The rate of AC scales sublinearly with the concentration of $4 \mu \mathrm{m}$ microspheres, and appears to follow a power law with an exponent $(2 / 3)$ as shown in Figure $4 d$

$$
\mathrm{AC}=\delta \sigma^{2 / 3} t
$$

where " $\delta$ " is the sticking probability and " $\sigma$ " is the volume fraction of the $4 \mu \mathrm{m}$ microspheres. As the concentration of the $4 \mu \mathrm{m}$ microspheres is successively halved, the slope decreases to $(0.5)^{2 / 3}=0.63$ and $(0.25)^{2 / 3}=0.40$, respectively (Figure $4 d)$. The exponent $(2 / 3)$ can be understood by approximating the entire deposited mass to be circular and of a radius " $R$ ", such that the volume and area coverage of the deposited mass is

\section{$\mathrm{VOL} \propto R^{3}$ and $\mathrm{AC} \propto R^{2}$}

By equating the above two equations, the area coverage scales as

$$
\mathrm{AC}=\delta \sigma^{2 / 3} t \propto R^{2} \propto \mathrm{VOL}^{2 / 3}
$$

Accordingly, the volume of deposited mass is directly proportional to the concentration of the $4 \mu \mathrm{m}$ microspheres $(\mathrm{VOL} \propto \sigma)$. The deposited volume (VOL) can be assumed to be made up of only $10 \mu \mathrm{m}$ microspheres, as the volume of a single $10 \mu \mathrm{m}$ microsphere is more than 15 times that of a $4 \mu \mathrm{m}$ microsphere. Therefore, on average, each $4 \mu \mathrm{m}$ microsphere facilitates the deposition of one more $10 \mu \mathrm{m}$ microsphere. The multi-microsphere system is unstable in $0.1 \mathrm{M} \mathrm{PBS}$ and forms clumps, rendering the deposition process erratic (Video S7).

To understand the deposition process in the multimicrosphere system, we closely examined the deposition of first few mcrospheres. At an initial flow rate of $10 \mu \mathrm{L} / \mathrm{min}$, microspheres of size $10 \mu \mathrm{m}$ pin on the microstructures. The rapid deposition then commences at a flow rate of $70 \mu \mathrm{L} / \mathrm{min}$ (Video S5), when the $4 \mu \mathrm{m}$ microspheres pin over the existing $10 \mu \mathrm{m}$ microspheres. As seen in Video S6 and Figure 5a, the preferred pinning sites of the $4 \mu \mathrm{m}$ microspheres are positions 

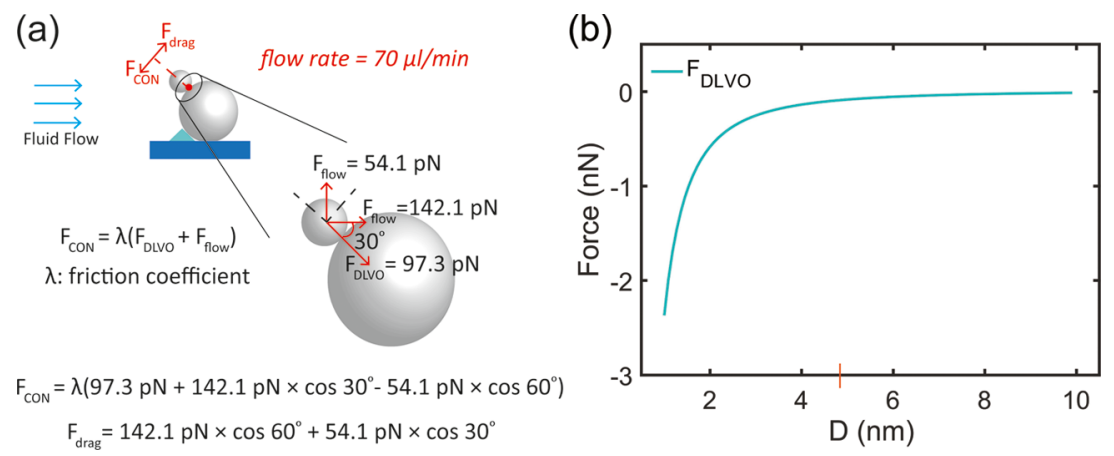

Figure 6. Contact force between the 4 and $10 \mu \mathrm{m}$ microspheres in DI water. (a) Free body diagram displaying the components of contact force on a $4 \mu \mathrm{m}$ microsphere pinned over a $10 \mu \mathrm{m}$ microsphere. (b) DLVO force between microspheres of size 4 and $10 \mu \mathrm{m}$, respectively.

3 and 4 (Figure $5 \mathrm{~b}$ ). The drag force, as estimated from hydrodynamic simulation (Section $\mathrm{S} 2$ ), is maximum at position $3\left(\sim 30-60^{\circ}\right.$ from horizontal) and position $4\left(90^{\circ}\right.$ from horizontal), which is undesirable for pinning (Figure $5 \mathrm{~b}$ ). However, the preference for positions 3 and 4 suggests that the pinning of the $4 \mu \mathrm{m}$ microspheres is dominated by the initial contact. Further, for a stable deposition, the contact force between the rough 4 and $10 \mu \mathrm{m}$ microspheres should be at least $\sim 140 \mathrm{pN}$ (Figure $5 \mathrm{c}$ ). The deposition process further proceeds with the $10 \mu \mathrm{m}$ microsphere gliding its way toward the posterior region (Scheme 1 in Figure 5c) after initial contact with the $4 \mu \mathrm{m}$ microspheres (Video S6). The drag force on the $10 \mu \mathrm{m}$ microspheres near the top is $\sim 1.25 \mathrm{nN}$ (Scheme 2, Figure 5d) and drops to $\sim 0.49 \mathrm{nN}$ (Scheme 1, Figure $5 \mathrm{~d}$ ) in the posterior region where the microsphere eventually settles. Even though the flow field always pushes the particles in the posterior region (Figure $5 \mathrm{~d}, \mathrm{e}$ ), the $4 \mu \mathrm{m}$ microspheres pin at the point of contact, whereas the $10 \mu \mathrm{m}$ microspheres follow the flow direction and deposit on the posterior side.

Components of Contact Force. The rapid deposition of multi-microspheres $(4+10 \mu \mathrm{m})$ over microstructures is a twostep process. Initially, $10 \mu \mathrm{m}$ microspheres pin on the microstructure at a flow rate of $10 \mu \mathrm{L} / \mathrm{min}$, which is followed by large-scale accumulation at a higher flow rate of $70 \mu \mathrm{L} / \mathrm{min}$. The rapid deposition proceeds with the pinning of a $4 \mu \mathrm{m}$ microsphere on an already pinned $10 \mu \mathrm{m}$ microsphere (Figure 5a). For stable pinning of a $4 \mu \mathrm{m}$ microsphere, the contact force $\left(F_{\text {con }}\right)$ should balance the drag force $\left(F_{\text {drag }}\right)$ as shown in Figure 6a. The contact force can be expressed as the net friction force due to the attractive DLVO interaction and drag generated by the fluid flow

$$
F_{\text {con }}=\lambda\left(F_{\text {DLVO }}+F_{\text {flow }}\right)
$$

where " $\lambda$ " is the friction coefficient. The minimum gap at which $4 \mu \mathrm{m}$ microspheres can graze over $10 \mu \mathrm{m}$ microspheres is limited by its roughness $(\sim 4.7 \mathrm{~nm})$. The attractive DLVO force between the 4 and $10 \mu \mathrm{m}$ microspheres at a gap of 4.7 $\mathrm{nm}$ is $97.3 \mathrm{pN}$ (Figure 6b). Similarly, the drag force generated by the fluid in parallel and perpendicular directions of the flow is numerically estimated as 142.1 and $54.1 \mathrm{pN}$, respectively (Section S2). The net friction force calculated by adding the components of DLVO and drag force perpendicular to the line of contact (Figure 6a) is

$$
\begin{aligned}
F_{\text {con }}= & \lambda\left(97.3 \mathrm{pN}+142.1 \mathrm{pN} \times \cos 30^{\circ}-54.1 \mathrm{pN}\right. \\
& \left.\times \cos 60^{\circ}\right) \\
= & \lambda \times 193.31 \mathrm{pN}
\end{aligned}
$$

The drag force opposite to the direction of contact force is estimated by adding the flow components parallel to the line of contact

$$
\begin{aligned}
F_{\text {drag }} & =142.1 \mathrm{pN} \times \cos 60^{\circ}+54.1 \mathrm{pN} \times \cos 30^{\circ} \\
& =117.9 \mathrm{pN}
\end{aligned}
$$

Accordingly, the minimum friction coefficient needed to balance the drag force is $\lambda=F_{\text {drag }} /\left(F_{\text {con }}=0.6\right)$. The estimated friction coefficient $\lambda$ is of similar order as reported for the roughness in the range of $\sim 10 \mathrm{~nm} .{ }^{8,45}$ The DLVO force for the $4 \mu \mathrm{m}$ microsphere of lower roughness $\left(R_{\mathrm{a}}=1.5 \mathrm{~nm}\right)$ while grazing on the $10 \mu \mathrm{m}$ microsphere, i.e., at the gap of $1.5 \mathrm{~nm}$, is $993 \mathrm{pN}$. The total contact force perpendicular to the line of contact would be $1089 \mathrm{pN}$, which is significantly higher than the former case. However, if the friction coefficient drops to say $\lambda=0.1$, owing to lower roughness, then the contact force $\left(F_{\text {con }}=\lambda \times 1089 \mathrm{pN}=108.9 \mathrm{pN}\right)$ would not be able to balance the drag force $\left(F_{\mathrm{drag}}=117.9 \mathrm{pN}\right)$ due to fluid flow. The friction coefficient is proportional to the roughness $\left(R_{\mathrm{a}}\right)$ but requires specialized tribometric measurements for accurate quantification. ${ }^{46-48}$ Nevertheless, the experimental evidence suggests robust pinning of $4 \mu \mathrm{m}$ microspheres of higher roughness leading to rapid deposition.

\section{CONCLUSIONS}

To summarize, the deposition process is initiated by the pinning of $10 \mu \mathrm{m}$ microspheres on the downslope region when flown $(10 \mu \mathrm{L} / \mathrm{min})$ over wedge-shaped symmetric microstructures of height $1.5 \mu \mathrm{m}$ and width $6 \mu \mathrm{m}$. The incoming microspheres then interact with the pinned microspheres through an attractive DLVO and roughness mediated friction force. The electrostatic component of the DLVO force can be decreased by introducing a salt solution such as PBS buffer $(0.1 \mathrm{M})$, which leads to the formation of linear chains (Figures $7 \mathrm{a}$ and $3 \mathrm{a})$. Alternatively, in DI water and at higher flow rates (70 $\mu \mathrm{L} / \mathrm{min})$, the presence of $4 \mu \mathrm{m}$ microspheres of roughness $R_{\mathrm{a}}=4.7 \mathrm{~nm}$ acts as a bridge between the incoming $10 \mu \mathrm{m}$ microspheres and already pinned $10 \mu \mathrm{m}$ microspheres (Figures $7 \mathrm{~b}$ and $5 \mathrm{a}$ ), resulting in the rapid deposition. Interestingly, a slight drop in the roughness of $4 \mu \mathrm{m}$ microspheres from 4.7 to $1.5 \mathrm{~nm}$ does not lead to rapid deposition. These findings put 
(a)

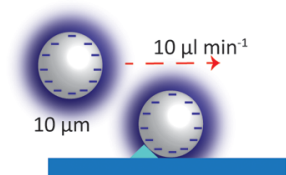

DI: Debye length $\approx 950 \mathrm{~nm}$

(b)

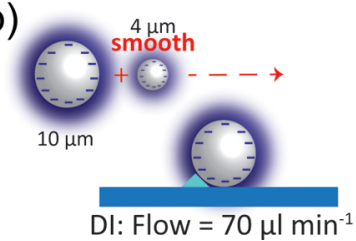

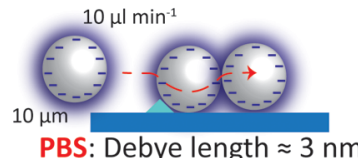

PBS: Debye length $\approx 3 \mathrm{~nm}$

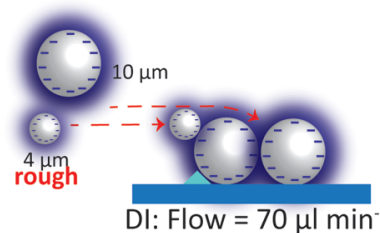

Figure 7. Infographic showing route toward the deposition process. (a) Screening of the electric double layer repulsion by using PBS enhances the probability of contact between microspheres, thereby assisting the formation of linear chains at a flow rate of $10 \mu \mathrm{L} / \mathrm{min}$. (b) Rougher $4 \mu \mathrm{m}$ microspheres at a high flow rate of $70 \mu \mathrm{L} / \mathrm{min}$ pin onto $10 \mu \mathrm{m}$ microspheres, initiating the rapid deposition of the incoming $10 \mu \mathrm{m}$ microsphere.

bounds on the required ionic strength and roughness that can lead to rapid colloidal deposition in microfluidic channels.

\section{ASSOCIATED CONTENT}

\section{SI Supporting Information}

The Supporting Information is available free of charge at https://pubs.acs.org/doi/10.1021/acs.langmuir.1c00370.

Pinning of $10 \mu \mathrm{m}$ microspheres in DI water medium; hydrodynamic equations for COMSOL simulation; DLVO force calculation; analytical expression of drag force near a surface; materials and methods; mixture of $10 \mu \mathrm{m}$ microspheres and microspheres of lower roughness $\left(R_{\mathrm{a}}=1.5 \mathrm{~nm}\right)(\mathrm{PDF})$

Pinning of $10 \mu \mathrm{m}$ microspheres on the downslope at a flow rate of $10 \mu \mathrm{L} / \mathrm{min}$ (Video S1) (AVI)

Pinning of $10 \mu \mathrm{m}$ microspheres on both the upslope and downslope at a flow rate of $0.1 \mu \mathrm{L} / \mathrm{min}$ (Video S2) (AVI)

Microsphere of size $10 \mu \mathrm{m}$ in PBS $0.1 \mathrm{M}$ forms linear chain when passed at a flow rate of $10 \mu \mathrm{L} / \mathrm{min}$ (Video S3) (AVI)

Longer version of Video S3 to show coupling in nearby chains (Video S4) (AVI)

Microsphere of size 10 and $4 \mu \mathrm{m}$ (mixed) in DI water deposits rapidly when passed at an initial flow rate of 10 $\mu \mathrm{L} / \mathrm{min}$ and later increased to $70 \mu \mathrm{L} / \mathrm{min}$ (Video S5) (AVI)

Deposition of first few microspheres in the case of rapid deposition as shown in Video S5 (Video S6) (AVI) Microsphere of size 10 and $4 \mu \mathrm{m}$ (mixed) in PBS $0.1 \mathrm{M}$ aggregate to form clumps (Video S7) (AVI)

\section{AUTHOR INFORMATION}

\section{Corresponding Authors}

P. Prakash - Centre for Nanoscience and Engineering, Indian Institute of Science, Bangalore 560012, India; Present Address: Departmentof Applied Mathematics and Theoretical Physics, University of Cambridge, Wilberforce Road, Cambridge CB3 0WA, United Kingdom.; 이 orcid.org/0000-0002-5282-0807; Email: pp467@ cam.ac.uk

M. Varma - Centre for Nanoscience and Engineering, Indian Institute of Science, Bangalore 560012, India; Robert Bosch Centre for Cyber Physical Systems, Indian Institute of Science, Bangalore 560012, India; Email: mvarma@iisc.ac.in

\section{Author}

A. Z. Abdulla - Department of Physics, Indian Institute of Science, Bangalore 560012, India; Present

Address: Laboratoire de Biologie et Modelisation de la Cellule, ENS de Lyon, 69364 Lyon, Cedex 07, France.

Complete contact information is available at: https://pubs.acs.org/10.1021/acs.langmuir.1c00370

\section{Notes}

The authors declare no competing financial interest.

\section{ACKNOWLEDGMENTS}

P.P. acknowledges financial support from the INSPIRE fellowship scheme of the Department of Science and Technology, Government of India. We thank the anonymous reviewers for their insightful comments that have significantly improved our manuscript.

\section{REFERENCES}

(1) Dressaire, E.; Sauret, A. Clogging of Microfluidic Systems. Soft Matter 2017, 13, 37-48.

(2) Radhakrishnan, A. N. P.; Marques, M. P. C.; Davies, M. J.; O'Sullivan, B.; Bracewell, D. G.; Szita, N. Flocculation on a Chip: A Novel Screening Approach to Determine Floc Growth Rates and Select Flocculating Agents. Lab Chip 2018, 18, 585-594.

(3) Donev, A.; Torquato, S.; Stillinger, F. H.; Connelly, R. Jamming in Hard Sphere and Disk Packings. J. Appl. Phys. 2004, 95, 989-999.

(4) Palacci, J.; Sacanna, S.; Steinberg, A. P.; Pine, D. J.; Chaikin, P. M. Living Crystals of Light-Activated Colloidal Surfers. Science 2013, 339, 936-940.

(5) Singh, R.; Adhikari, R. Universal Hydrodynamic Mechanisms for Crystallization in Active Colloidal Suspensions. Phys. Rev. Lett. 2016, 117, No. 228002.

(6) Wang, G. R.; Yang, F.; Zhao, W. There Can Be Turbulence in Microfluidics at Low Reynolds Number. Lab Chip 2014, 14, 14521458.

(7) Mohtaschemi, M.; Puisto, A.; Illa, X.; Alava, M. J. Rheology Dynamics of Aggregating Colloidal Suspensions. Soft Matter 2014, 10, 2971-2981.

(8) Hsu, C. P.; Ramakrishna, S. N.; Zanini, M.; Spencer, N. D.; Isa, L. Roughness-Dependent Tribology Effects on Discontinuous Shear Thickening. Proc. Natl. Acad. Sci. U.S.A. 2018, 115, 5117-5122.

(9) Bacchin, P.; Derekx, Q.; Veyret, D.; Glucina, K.; Moulin, P. Clogging of Microporous Channels Networks: Role of Connectivity and Tortuosity. Microfluid. Nanofluid. 2014, 17, 85-96.

(10) Sendekie, Z. B.; Bacchin, P. Colloidal Jamming Dynamics in Microchannel Bottlenecks. Langmuir 2016, 32, 1478-1488.

(11) Robert De Saint Vincent, M.; Abkarian, M.; Tabuteau, H. Dynamics of Colloid Accumulation under Flow over Porous Obstacles. Soft Matter 2016, 12, 1041-1050.

(12) Marin, A.; Lhuissier, H.; Rossi, M.; Kähler, C. J. Clogging in Constricted Suspension Flows. Phys. Rev. E 2018, 97, No. 021102.

(13) Souzy, M.; Zuriguel, I.; Marin, A. Transition from Clogging to Continuous Flow in Constricted Particle Suspensions. Phys. Rev. E 2020, 101, No. 060901.

(14) Sauret, A.; Somszor, K.; Villermaux, E.; Dressaire, E. Growth of Clogs in Parallel Microchannels. Phys. Rev. Fluids 2018, 3, No. 104301.

(15) Yodh, J. S.; Spandan, V.; Mahadevan, L. Suspension Jams in a Leaky Microfluidic Channel. Phys. Rev. Lett. 2020, 125, No. 044501. 
(16) Reichhardt, C.; Reichhardt, C. J. O. Controlled Fluidization, Mobility, and Clogging in Obstacle Arrays Using Periodic Perturbations. Phys. Rev. Lett. 2018, 121, No. 068001.

(17) Zuriguel, I.; Parisi, D. R.; Hidalgo, R. C.; Lozano, C.; Janda, A.; Gago, P. A.; Peralta, J. P.; Ferrer, L. M.; Pugnaloni, L. A.; Clément, E.; et al. Clogging Transition of Many-Particle Systems Flowing through Bottlenecks. Sci. Rep. 2014, 4, No. 7324.

(18) Sauret, A.; Barney, E. C.; Perro, A.; Villermaux, E.; Stone, H. A.; Dressaire, E. Clogging by Sieving in Microchannels: Application to the Detection of Contaminants in Colloidal Suspensions. Appl. Phys. Lett. 2014, 105, No. 074101.

(19) Wyss, H. M.; Blair, D. L.; Morris, J. F.; Stone, H. A.; Weitz, D. A. Mechanism for Clogging of Microchannels. Phys. Rev. E 2006, 74, No. 061402 .

(20) Agbangla, G. C.; Bacchin, P.; Climent, E. Collective Dynamics of Flowing Colloids during Pore Clogging. Soft Matter 2014, 10, 6303-6315.

(21) Ortiz, C. P.; Daniels, K. E.; Riehn, R. Nonlinear Elasticity of Microsphere Heaps. Phys. Rev. E 2014, 90, No. 022304.

(22) Henry, C.; Minier, J. P.; Lefêvre, G. Towards a Description of Particulate Fouling: From Single Particle Deposition to Clogging. Adv. Colloid Interface Sci. 2012, 185-186, 34-76.

(23) Bizmark, N.; Schneider, J.; Priestley, R. D.; Datta, S. S. Multiscale Dynamics of Colloidal Deposition and Erosion in Porous Media. Sci. Adv. 2020, 6, 2530-2543.

(24) Ramachandran, V.; Fogler, H. S. Multilayer Deposition of Stable Colloidal Particles during Flow within Cylindrical Pores. Langmuir 1998, 14, 4435-4444.

(25) Cejas, C. M.; Monti, F.; Truchet, M.; Burnouf, J. P.; Tabeling, P. Particle Deposition Kinetics of Colloidal Suspensions in Microchannels at High Ionic Strength. Langmuir 2017, 33, 6471-6480.

(26) Prakash, P.; Varma, M. Trapping/Pinning of Colloidal Microspheres over Glass Substrate Using Surface Features. Sci. Rep. 2017, 7, No. 15754.

(27) Clavaud, C.; Be-ruta, A.; Metzgera, B.; Forterrea, Y. Revealing the Frictional Transition in Shear-Thickening Suspensions. Proc. Natl. Acad. Sci. U.S.A. 2017, 114, 5147-5152.

(28) Burke, A. P.; Farb, A.; Malcom, G. T.; Liang, Y.; Smialek, J.; Virmani, R. Coronary Risk Factors and Plaque Morphology in Men with Coronary Disease Who Died Suddenly. N. Engl. J. Med. 1997, 336, 1276-1282.

(29) Libby, P.; Ridker, P. M.; Hansson, G. K. Progress and Challenges in Translating the Biology of Atherosclerosis. Nature 2011, 473, 317-325.

(30) Kojima, Y.; Volkmer, J. P.; McKenna, K.; Civelek, M.; Lusis, A. J.; Miller, C. L.; Direnzo, D.; Nanda, V.; Ye, J.; Connolly, A. J.; et al. CD47-Blocking Antibodies Restore Phagocytosis and Prevent Atherosclerosis. Nature 2016, 536, 86-90.

(31) Hajhosseiny, R.; Bahaei, T. S.; Prieto, C.; Botnar, R. M. Molecular and Nonmolecular Magnetic Resonance Coronary and Carotid Imaging. Arterioscler., Thromb., Vasc. Biol. 2019, 39, 569-582.

(32) Prakash, P.; Pahal, S.; Varma, M. Fluorescence Recovery after Photobleaching in Ultrathin Polymer Films. Macromol. Chem. Phys. 2018, 219, No. 1700543.

(33) Israelachvili, J. N. Intermolecular and Surface Forces, 3rd ed.; Elsevier Inc., 2011.

(34) Bhattacharjee, S.; Ko, C.-H.; Elimelech, M. DLVO Interaction between Rough Surfaces. Langmuir 1998, 14, 3365-3375.

(35) Kligerman, Y.; Varenberg, M. Elimination of Stick-Slip Motion in Sliding of Split or Rough Surface. Tribol. Lett. 2014, 53, 395-399.

(36) Lee, D. W.; Banquy, X.; Israelachvili, J. N. Stick-Slip Friction and Wear of Articular Joints. Proc. Natl. Acad. Sci. U.S.A. 2013, 110, E567-E574.

(37) Thompson, P. A.; Robbins, M. O. Origin of Stick-Slip Motion in Boundary Lubrication. Science 1990, 250, 792-794.

(38) Bengisu, M. T.; Akay, A. Stick-Slip Oscillations: Dynamics of Friction and Surface Roughness. J. Acoust. Soc. Am. 1999, 105, 194205.
(39) Chen, Z.; Khajeh, A.; Martini, A.; Kim, S. H. Chemical and Physical Origins of Friction on Surfaces with Atomic Steps. Sci. Adv. 2019, 5, No. eaaw0513.

(40) Mulvaney, S. P.; Cole, C. L.; Kniller, M. D.; Malito, M.; Tamanaha, C. R.; Rife, J. C.; Stanton, M. W.; Whitman, L. J. Rapid, Femtomolar Bioassays in Complex Matrices Combining Microfluidics and Magnetoelectronics. Biosens. Bioelectron. 2007, 23, 191-200.

(41) Gijs, M. A. M.; Lacharme, F.; Lehmann, U. Microfluidic Applications of Magnetic Particles for Biological Analysis and Catalysis. Chem. Rev. 2010, 110, 1518-1563.

(42) White, F. M. Fluid Mechanics, 8th ed.; Mc-Graw Hill, 2016.

(43) Prakash, P.; Abdulla, A. Z.; Singh, V.; Varma, M. Tuning the Torque-Speed Characteristics of the Bacterial Flagellar Motor to Enhance Swimming Speed. Phys. Rev. E 2019, 100, No. 062609.

(44) Prakash, P.; Abdulla, A. Z.; Singh, V.; Varma, M. Swimming Statistics of Cargo-Loaded Single Bacteria. Soft Matter 2020, 16, 9499-9505.

(45) Zhang, Y.; Sundararajan, S. The Effect of Autocorrelation Length on the Real Area of Contact and Friction Behavior of Rough Surfaces. J. Appl. Phys. 2005, 97, No. 103526.

(46) Berman, A. D.; Ducker, W. A.; Israelachvili, J. N. Origin and Characterization of Different Stick-Slip Friction Mechanisms. Langmuir 1996, 12, 4559-4562.

(47) Fernandez, N.; Cayer-Barrioz, J.; Isa, L.; Spencer, N. D. Direct, Robust Technique for the Measurement of Friction between Microspheres. Langmuir 2015, 31, 8809-8817.

(48) Zhang, Y.; Sundararajan, S. Adhesion and Friction Studies of Silicon Surfaces Processed Using a Microparticle-Based Method. Tribol. Lett. 2006, 23, 1-5. 